\title{
Genetics of Salt and Cold Tolerance in Tomato: Quantitative Analysis and QTL Mapping
}

\author{
Majid R. Foolad \\ Department of Horticulture, The Pennsylvania State University, University Park, PA 16802 USA \\ (Correspondence; FAX, 814-863-6139; E-mail,
}

\begin{abstract}
Quantitative genetic analysis of tomato response to salt or cold stress during seed germination and vegetative growth indicated that both salt and cold tolerance were complex traits, controlled by more than one gene and highly influenced by environmental variation. Molecular marker analyses indicated that at each stage of plant development salt tolerance or cold tolerance was generally controlled by the effects of a few major QTLs (quantitative trait loci) which acted in concert with a number of smallereffect QTLs. At the seed germination stage, two types of QTLs were identified: those which affected germination under both stress and nonstress conditions, and thus were called stress-nonspecific QTLs, and those which contributed to rapid seed germination only under specific stress conditions, and thus were called stress-specific QTLs. Generally, the stress-nonspecific QTLs exhibited larger effects than the stress-specific QTLs. Consistent with this observation, selection for either salt or cold tolerance during germination resulted in progeny with improved germination under salt and cold stress as well as nonstress conditions. Comparison of salt tolerance during germination and vegetative growth indicated that mostly different QTLs contributed to tolerance at these two developmental stages; furthermore, selection for salt tolerance during germination did not affect progeny salt tolerance during vegetative growth. Similar results were obtained when cold tolerance during germination and vegetative growth were compared. The overall results indicate that, in tomato, stress tolerance during germination is independent of stress tolerance during vegetative growth. However, simultaneous improvement of plants for stress tolerance at multiple stages of plant development should be feasible through marker-assisted selection and breeding.
\end{abstract}

\section{Introduction}

With the rapid increase in human population, the world faces a greater demand on agricultural output than at any time in history. During the past 50 years, agricultural productivity for major crop species in the US has, on average, doubled [1]. Fifty percent or more of this increase has resulted from crop improvement through plant breeding, and the rest due to the cultivation of more land, greater farming inputs (e.g. fertilizers, pesticides and water) and advancements in agricultural technology. In the future, however, the contribution of plant breeding to increased food production will have to be greater because of the limitations in nongenetic approaches to increase agricultural production. Examples of the latter are the shrinking natural resources (e.g. fresh water and petroleum) and lack of additional arable land. Thus, more sophisticated breeding strategies based on genetics and molecular biology in addition to breeders ingenuity and experience are needed. Furthermore, previous plant breeding efforts focused mainly on the development of cultivars with high yield potential in normal or highly favored environments (e.g., optimal amount of water and nutrients). However, current crop production is largely limited by environmental stresses. For example, it is estimated that only $10 \%$ of the world's arable land may be categorized as free of stress [2]. Extreme temperatures, drought, soil salinity and nutrient imbalances are among the major environmental constraints to crop production.
In the future, to fulfill the need for greater food production, extensive efforts have to be made in developing plants which are capable of producing stable and economic yields in stress environments.

Significant progress in breeding for stress tolerance depends on an understanding of the genetic and epigenetic dimensions of gene action and the physiology of stress tolerance. Although considerable information is presently available regarding the physiological and metabolic aspects of plant response to environmental stresses [3-5], effort devoted to the characterization of the genetic control of stress tolerance has been limited [6-9].

Previous investigations indicated that plant response to environmental stress is complex, controlled by more than one gene and highly influenced by environmental variation $[6,10,11]$. In addition, stress tolerance is a developmentallyregulated, stage-specific phenomenon; tolerance at one stage of plant development may not be correlated with tolerance at other developmental stages [12-14]. Thus, specific ontogenetic stages of the plant such as germination and emergence, seedling survival and growth, and vegetative growth and reproduction, should be evaluated separately for assessment of tolerance and the identification of useful genetic components. Partitioning of stress tolerance into component traits related to specific ontogenetic stages would contribute to a better understanding of the genetic control of stress tolerance and thus, may facilitate rapid development of stress tolerant plants.

During the past several decades, theories of Men- 
delian genetics have been useful for discerning the genetic bases of simply inherited (qualitative) traits; such traits are often controlled by one or few genes with distinguishable and discrete effects and their expression is minimally affected by environmental variation. Because of their simple inheritance, considerable breeding progress has been made for qualitative traits. However, most agriculturally important traits, including environmental stress tolerance, exhibit continuous variation; such traits are often conditioned by the effects of several (and some times many) genetic loci which interact with each other and with the environment. Although the development of biometrical genetic models during the early part of the 20th century facilitated the investigation of quantitative traits $[15,16]$, such protocols have been less efficient in discerning the precise genetic bases of quantitative traits and hence, contributed minimally to the genetic improvement of crop plants. Thus, researchers have continuously searched for alternative approaches for the improvement of plants for traits with complex inheritance.

Recent advances in molecular techniques have facilitated a better understanding of the genetic and biochemical bases of many important agricultural characteristics and enabled plant breeders to make changes in many traits by design rather than by accident. Although most molecular protocols, such as gene cloning and transformation, have thus far been useful only for the characterization and genetic manipulation of simply inherited traits, some techniques, such as the use of molecular markers, have been beneficial for the refinement of conventional protocols of dealing with quantitative traits. For example, molecular marker technology has facilitated the identification and characterization of chromosomal regions (often called quantitative trait loci or QTLs) which control the expression of quantitative traits. This technology has facilitated the identification of the number and magnitude of effects of QTLs, the detection of intralocus and interlocus interactions, and a better understanding of important genetic phenomena such as heterosis, transgressive segregation and genotype $\times$ environment interactions [1719]. Within the last two decades, QTL mapping techniques have contributed to a greater understanding of the genetic bases of many agriculturally and biologically important quantitative traits such as yield, resistance/tolerance to biotic and abiotic stresses, and nutritional quality in numerous crop species [20-22].

A thrust of our research program has been the genetic characterization of plant response to two environmental stresses, namely salt stress and cold stress, and the use of the knowledge to develop germplasm with improved salt tolerance and cold tolerance. We have chosen to employ both the conventional protocols of biometrical genetics and the contemporary techniques of molecular mapping and QTL analysis for the characterization of the genetic bases of stress tolerance in tomato. Our investigations have resulted in the identification of genes (QTLs) controlling salt tolerance and cold tolerance, determination of genetic relationships between stress tolerance at different developmental stages, and determination of genetic relationships between tolerance to different environmen- tal stresses. In addition, we have investigated genetic relationships between plant performance in stress and nonstress environments. In the following paragraphs, I summarize the results of some of our investigations, focusing on the use of molecular marker technology in discerning the genetic bases of stress tolerance.

\section{Genetics of salt tolerance during seed germination}

The ability of the seed to germinate rapidly and uniformly under salt stress is a desirable trait for many direct-seeded crops. In many crop species including tomato, Lycopersicon esculentum Mill., seed germination and early seedling growth are the most sensitive stages to salinity stress $[23,24]$. Salinity stress greatly delays the onset, reduces the rate and increases the dispersion of germination events. Development of cultivars with the ability to germinate rapidly and emerge reliably from saline-affected soils would contribute significantly to the efficiency of stand establishment.

Modern cultivars of tomato are highly sensitive to salt stress during seed germination, however, genetic sources of salt tolerance have been identified among the related wild species and primitive cultivars of tomato $[25,26]$. Investigations of some of these resources indicated that salt tolerance during germination in tomato was genetically controlled with additivity being the major genetic component $[27,28]$. Partitioning of the genetic variation into those attributable to the effects of different seed tissues including embryo, endosperm and testa (seed coat) indicated that a great portion of the variation for salt tolerance was due to the additive effects of the seed endosperm $[27,29]$. Further investigation indicated that salt tolerance during seed germination in tomato could be improved by phenotypic selection and a realized heritability of ca. 0.73 was obtained for this trait [11]. However, from these studies the number and individual effects of contributing genetic factors could not be determined.

Two QTL mapping approaches were employed to identify the number, chromosomal location and magnitude of direct and interactive effects of QTLs contributing to salt tolerance during seed germination. First, a traitbased marker analysis (TBA) of an $F_{2}$ population of a cross between a salt sensitive tomato breeding line (UCT5) and a germination salt tolerant accession (LA716) of a green-fruited tomato wild species, Lycopersicon pennellii (Corr.) D'Arcy, resulted in the identification of eight QTLs on seven chromosomes with significant effects on this trait [30, 31]. In this study, it was also determined that the same QTLs contributed to salt tolerance at different salt stress levels, and thus, selection for salt tolerance at one stress level would result in progeny with improved salt tolerance at a wide range of salt stress levels.

The second QTL mapping approach was a markerbased analysis (MBA) of a $\mathrm{BC}_{1} \mathrm{~S}_{1}$ population of a cross between a salt sensitive tomato breeding line (NC84173) and a salt tolerant accession (LA722) of a red-fruited tomato wild species, Lycopersicon pimpinellifolium (Jusl.) Mill. (see Fig. 1A for the germination performance of the parental and $\mathrm{BC}_{1} \mathrm{~S}_{1}$ progeny under salt stress). This anal- 

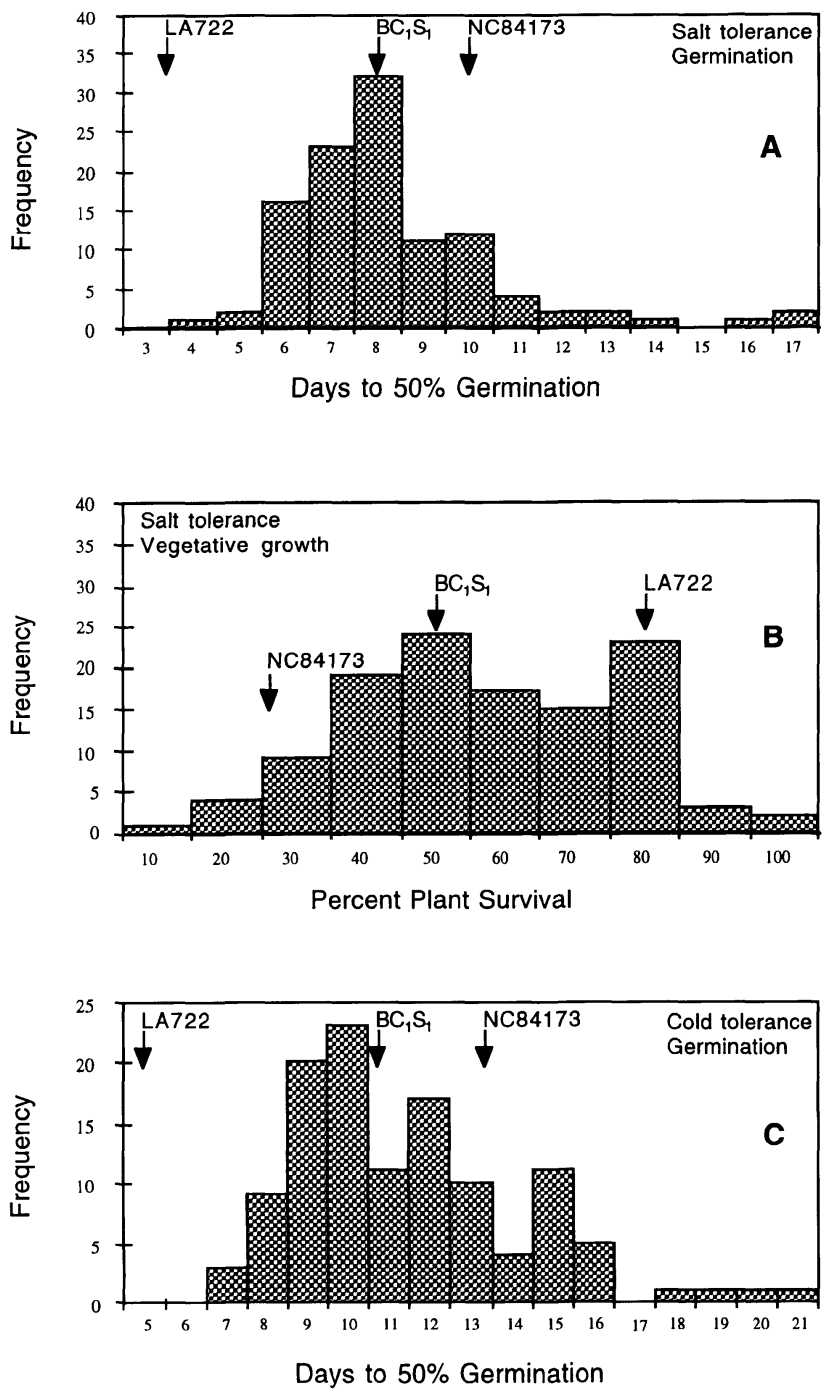

Fig. 1 Trait distributions for salt tolerance during germination (A) and vegetative growth (B) and cold tolerance during germination $(C)$ in the $\mathrm{BC}_{1} \mathrm{~S}_{1}$ population of a cross between Lycopersicon esculentum (NC84173) and L. pimpinellifolium (LA722). Means of the parental lines and $\mathrm{BC}_{1} \mathrm{~S}_{1}$ population are shown by arrows. Note that the distributions are on different scales.

ysis identified seven QTLs on six chromosomes with significant effects on salt tolerance during germination [32] (Table 1, Fig. 2). In this study, both the interval mapping, using MAPMAKER/QTL computer program v. 3.0 [33], and the single-marker analysis, using QGENE computer program [34], revealed similar results (Table 1). The individual effects of the QTLs ranged from 6.5 to $15.6 \%$ with cumulative effects of up to $44.5 \%$ of the total phenotypic variation for this trait. Several pairwise epistatic interactions were identified between QTL-linked and QTL-unlinked markers as well as among QTLunlinked markers. However, the number (percentage) of significant two-locus interactions was smaller than the percentage expected to occur by chance, and thus, it is likely that the identified interactions were due to chance effects.

The comparison between the results of the two QTL mapping studies indicated that while some of the identified
QTLs were conserved across tomato wild species, there were some species-specific QTLs for salt tolerance during germination. Thus, it may be possible to improve salt tolerance of the cultivated tomato at the germination stage by pyramiding salt tolerance genes introduced from different related wild species through marker-assisted selection and breeding.

\section{Genetics of salt tolerance during vegetative growth}

Most crop plants are sensitive to low to moderate levels of salt in the soil during vegetative growth [23]. Regardless of the cause (ion toxicity, water deficit and/or nutrient ion imbalance), salt stress severely impedes normal plant growth and development, resulting in reduced crop productivity or total crop loss. In tomato, potential sources of salt tolerance during vegetative growth have been identified among the related wild species and primitive cultivars [7, 35-37]. These genetic resources could potentially be utilized to enhance salt tolerance of modern cultivars. However, insufficient genetic knowledge of the tolerance-related traits and the lack of efficient selection criteria have restricted breeding efforts. We have initiated a research in tomato to discern the genetic basis of salt tolerance during vegetative growth, identify morphological, physiological and genetic markers associated with tolerance, and transfer tolerance components into modern cultivars through marker-assisted selection.

In an initial effort to learn more about the inheritance of salt tolerance during vegetative growth, a salt sensitive tomato breeding line (UCT5) and a salt tolerant primitive cultivar (PI174263) and their $\mathrm{F}_{1}, \mathrm{~F}_{2}$ and $\mathrm{BC}_{1}$ progeny were evaluated for vegetative growth in two treatments of control (no salt added) and salt stress using aerated hydroponic systems. The results indicated that both absolute salt tolerance (i.e., absolute growth under salt stress) and relative (physiological) salt tolerance (i.e., growth under salt stress relative to growth under nonstress) were under genetic control with simple (additive and dominance) genetic effects explaining most of the variation; nonallelic interactions, although significant, were far less important [7]. Physiological evaluation of the parental and progeny generations indicated that under salt stress leaves of PI174263 accumulated significantly less $\mathrm{Na}^{+}$and $\mathrm{Cl}^{-}$and more $\mathrm{Ca}^{2+}$ than leaves of UCT5, and that growth under salt stress was positively correlated with leaf $\mathrm{Ca}^{2+}$ content and negatively correlated with leaf $\mathrm{Na}^{+}$content [8]. Generation means analysis (GMA) indicated that under salt stress both $\mathrm{Na}^{+}$and $\mathrm{Ca}^{2+}$ accumulations in the leaf were genetically controlled with additivity being the major genetic component. The overall results indicated that the inherent genetic capabilities of PI174263 to maintain high tissue $\mathrm{Ca}^{2+}$ levels and to exclude $\mathrm{Na}^{+}$from the shoot were essential features underlying its adaptation to salt stress and that these features were highly heritable. Thus, tissue ion concentration was suggested as a useful selection criterion when breeding for improved salt tolerance of tomato during vegetative growth [8].

To identify QTLs conferring salt tolerance during vegetative growth, a $\mathrm{BC}_{1} \mathrm{~S}_{1}$ population of an interspecific 


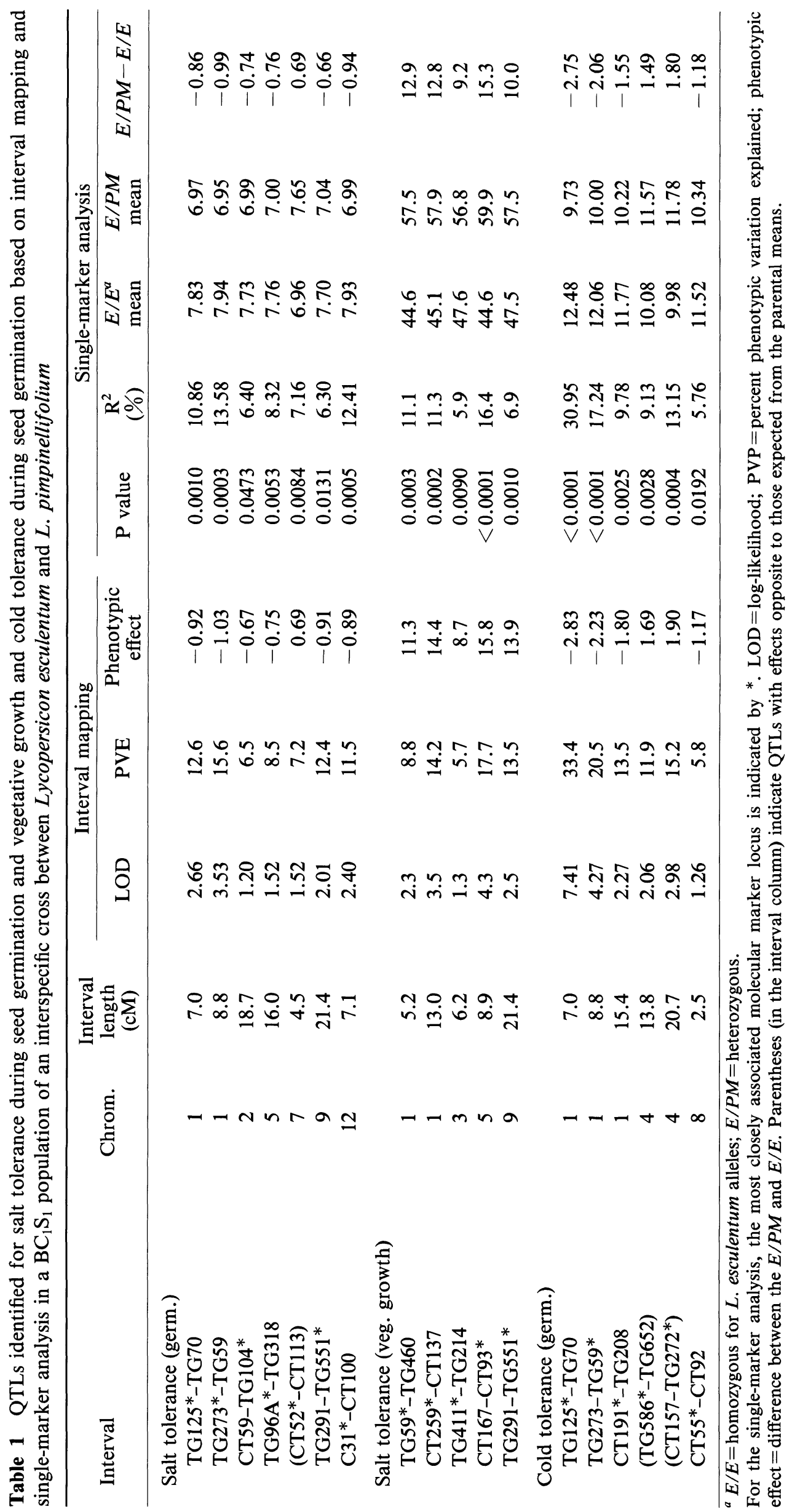


cross between NC84173 (salt sensitive) and the L. pimpinellifolium accession LA722 (salt tolerant) were evaluated for survival under salt stress [22]. Six to eight weeks old plants were evaluated for survival in aerated saline solution cultures with the salt concentration gradually raised to $700 \mathrm{mM} \mathrm{NaCl}+70 \mathrm{mM} \mathrm{CaCl} 2$ (equivalent to electrical conductivity of ca. $64 \mathrm{dS} / \mathrm{m}$ and water potential of ca. -35.2 bars). The two parental lines were distinctly different in salt tolerance: $80 \%$ of the LA722 plants versus $25 \%$ of the NC84173 plants survived for at least two weeks after the final salt concentration was reached (Fig. 1B). The $\mathrm{BC}_{1} \mathrm{~S}_{1}$ population exhibited continuous variation, typical of quantitative traits, with survival rate of the $\mathrm{BC}_{1} \mathrm{~S}_{1}$ families ranging from $9 \%$ to $94 \%$ with a mean of 51\% (Fig. 1B). Interval mapping and single-marker analysis identified five putative QTLs on four different chromosomes (Table 1, Fig. 2). The results were consistent with the previous suggestion that salt tolerance during vegetative growth in tomato was controlled by more than one gene [7, 8]. Each QTL accounted for between 5.7 and $17.7 \%$ of the total phenotypic variation with the combined effects (of all five QTLs) exceeding $46 \%$ of the variation. All QTLs had the positive QTL alleles (for salt tolerance) from the salt tolerant parent (LA722). Across QTLs, the effects were mainly additive in nature. Digenic epistatic interactions were evident among several QTL-linked and QTL-unlinked markers. However, similar to that observed for salt tolerance during germination, the number (percentage) of significant two-locus interactions was smaller than the percentage expected to occur by chance. The overall results indicate that tomato salt tolerance during vegetative growth is genetically controlled and could be improved by marker-assisted selection using interspecific variation [22].

\section{Relationship between salt tolerance during seed ger- mination and vegetative growth}

Three approaches were taken to determine whether the same genes contribute to salt tolerance during germination and vegetative growth in tomato. First, $\mathrm{F}_{4}$ progeny families of a cross between the breeding line UCT5 (salt sensitive at all stages of plant development) and the primitive cultivar PI174263 (salt tolerant during germination and vegetative growth) were evaluated for salt tolerance, in separate experiments, during germination and

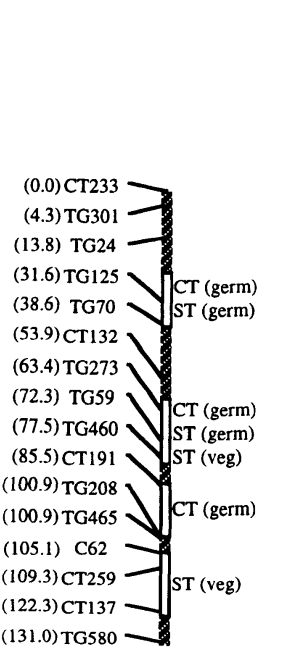

chr1

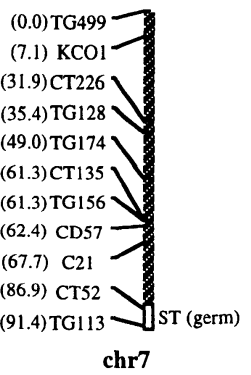

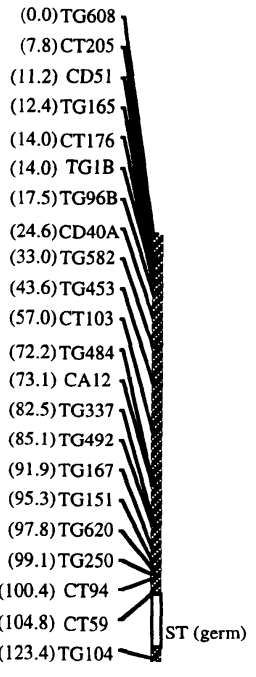

chr2

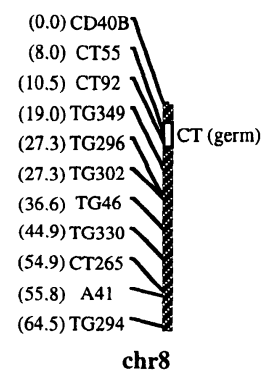

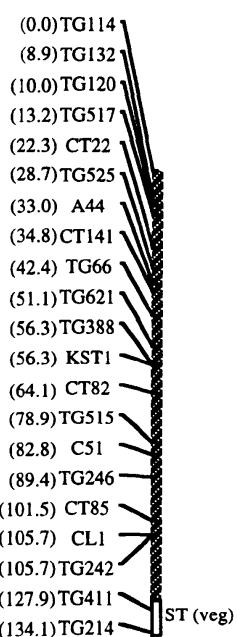

chr3

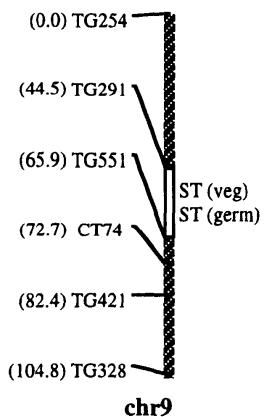

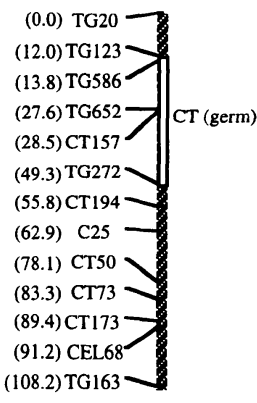

chr4

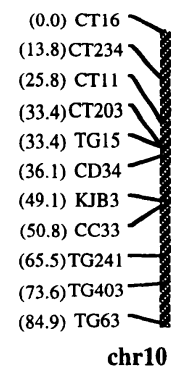

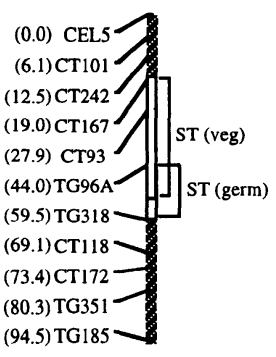

chr5

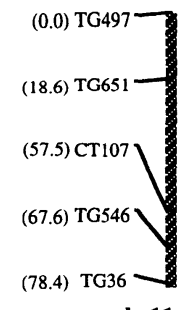

chr11

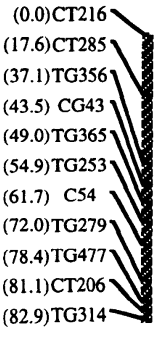

chr6

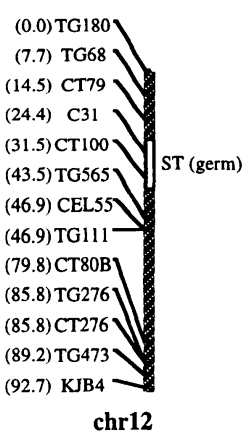

Fig. 2 A restriction fragment length polymorphism (RFLP) linkage map of tomato chromosomes constructed based on a $\mathrm{BC}_{1}$ population of a cross between Lycopersicon esculentum (NC84173; pistillate and recurrent parent) and L. pimpinellifolium (LA722). The chromosome number is shown at the bottom of each chromosome. The names and centiMorgan positions of the markers are listed at the left of chromosomes. The open boxes on chromosomes 1, 2, 3, 4, 5, 7, 8, 9 and 12 indicate the approximate location of QTLs identified for salt tolerance during germination (ST-germ) and vegetative stage (ST-veg) and cold tolerance during germination (CTgerm). 
vegetative growth. There were significant differences among the $\mathrm{F}_{4}$ families in terms of salt tolerance during germination and vegetative growth. However, there was no correlation between the ability of seeds to germinate rapidly and the ability of plants to grow under salt stress. The results indicated an absence of a phenotypic relationship between salt tolerance during germination and vegetative growth [14].

In the second approach, selection was made for rapid germination (the first $7.3 \%$ germinated) under salt stress in an $F_{2}$ population of the same cross and the selected $F_{3}$ progeny were evaluated for salt tolerance during both seed germination and vegetative growth. Selection for salt tolerance during germination significantly improved germination salt tolerance of the $\mathrm{F}_{3}$ progeny, indicating that the selection was effective. However, selection did not affect salt tolerance of the $F_{3}$ progeny during vegetative growth. Obviously, in these plant materials, genetic factors which contributed to salt tolerance during germination were different from those conferring salt tolerance during vegetative growth [14].

The third approach to determine the genetic relationship between salt tolerance during germination and vegetative growth involved the comparison of QTLs for salt tolerance at these two developmental stages. As described earlier, QTLs for these two traits were identified in a $\mathrm{BC}_{1} \mathrm{~S}_{1}$ population of a cross between $\mathrm{NC} 84173$ (salt sensitive during both stages) and $L$. pimpinellifolium accession LA722 (salt tolerant during both stages). Comparison of QTLs indicated that in most cases the locations of QTLs for salt tolerance during germination were different from the locations of QTLs for salt tolerance during vegetative growth; however, there were some coincidences in QTL locations (see Table 1 and Fig. 2) consistent with a small phenotypic correlation $(\mathrm{r}=-0.22$, $p<0.05$ ) observed between the rate of seed germination and the percentage of plant survival under salt stress in the $\mathrm{BC}_{1} \mathrm{~S}_{1}$ population.

The overall results indicate that salt tolerance during seed germination in tomato is generally independent of salt tolerance during vegetative growth. Therefore, when breeding for improved salt tolerance, each stage of plant development should be evaluated separately for assessment of tolerance and the identification of useful genetic components. However, the identification of QTLs for salt tolerance at both germination and vegetative growth stages should facilitate simultaneous introgression of markerlinked QTLs at both stages into desirable genetic backgrounds. Furthermore, the finding that only a few major QTLs could account for a large portion of the total phenotypic variation for salt tolerance at each stage indicates that marker-assisted selection for salt tolerance should be feasible, providing the opportunity to rapidly develop germplasm with enhanced salt tolerance at more than one stage of plant development.

\section{Genetics of cold tolerance during seed germination}

Germination rate of tomato seed decreases progressively as the temperature of the germination medium is decreased from 25 to $10^{\circ} \mathrm{C}$, and is inhibited below $10^{\circ} \mathrm{C}$ [38]. Low (chilling) temperatures $\left(15-10^{\circ} \mathrm{C}\right)$ significantly delay the onset, reduce the rate and increase the dispersion of germination events. Most commercial cultivars of tomato are sensitive to low temperatures during germination; however, considerable genetic variation exists within and among species of tomato for low temperature germination [38-40]. This variation could potentially be utilized to improve low temperature (cold) tolerance of commercial cultivars. Utilization of the genetic variation in breeding programs, however, requires knowledge of the genetic and epigenetic control of the trait. Unfortunately, efforts to characterize the genetic control of cold tolerance during germination in tomato have been limited.

We took three approaches to examine the genetic basis of cold tolerance during germination in tomato. First, a cold tolerant (PI120256) and a cold sensitive tomato cultivar (UCT5) and their reciprocal $F_{2}, F_{3}$ and $\mathbf{B C}_{1}$ progeny (total of 10 generations) were evaluated for germination at a low $\left(11 \pm 0.5^{\circ} \mathrm{C}\right)$ and a normal (control) temperature regime $\left(20 \pm 0.5^{\circ} \mathrm{C}\right)$. Weighted least square regression analysis indicated that under cold stress most of the variation in germination time was due to additive genetic effects; dominance and epistatic interactions were not significant [41]. Partitioning of the total genetic variance into those attributable to the effects of embryo, endosperm, testa and the cytoplasm indicated that additive effects of endosperm could account for $80 \%$ of the total genetic variance. The results were similar to those obtained for salt tolerance during germination in tomato [27].

The second approach to examining the genetic basis of cold tolerance during germination was the evaluation of response to phenotypic selection for improved germination under cold stress. In an $F_{2}$ population of a cross between UCT5 and PI120256, the fastest germinating seeds under low temperature $\left(11 \pm 0.5^{\circ} \mathrm{C}\right)$ were selected and the $\mathrm{F}_{3}$ progeny of the selected individuals were evaluated for germination under low temperature, and their performance compared with that of an unselected $\mathrm{F}_{3}$ population of the same cross. The results indicated that selection was highly effective and significantly improved germination performance of the progeny; a realized heritability of 0.74 was obtained for cold tolerance during germination [41]. From this investigation, we concluded that tomato seed germination under cold stress was genetically controlled with additivity being the major genetic component and that cold tolerance could be improved by phenotypic selection.

In the third approach, the number and chromosomal location of QTLs contributing to cold tolerance during germination were determined in an interspecific $\mathrm{BC}_{1} \mathrm{~S}_{1}$ population of a cross between the breeding line NC84173 (cold sensitive) and the L. pimpinellifolium accession LA722 (cold tolerant during germination). Two chromosomal locations (3-5 putative QTLs) with significant effects on cold tolerance germination were identified [42]. The $L$. pimpinellifolium accession had favorable QTL alleles (for cold tolerance) on chromosomes 1 and NC84173 had favorable QTL alleles on chromosome 4 (Table 1, Fig. 2). The percentage of phenotypic variation explained 
(PVE) by individual QTLs ranged from 11.9 to $33.4 \%$. Multilocus analysis indicated that the cumulative action of all significant QTLs accounted for $43.8 \%$ of the total phenotypic variance. Digenic epistatic interactions were evident only between two QTL-linked markers and two QTL-unlinked markers. Transgressive phenotypes were observed in the direction of cold sensitivity (see Fig. 1C).

The overall results indicate that cold tolerance during seed germination in tomato is mainly controlled by the effects of a few major QTLs. Thus, the prospect for improving this trait in tomato by marker-assisted introgression of QTLs from related wild species is good.

\section{Genetics of cold tolerance during vegetative growth}

Cold (chilling) tolerance during vegetative growth in tomato has been defined as the ability to resist damage below $\approx 10^{\circ} \mathrm{C}$ but above freezing temperature [43]. The cultivated tomato prefers a growth temperature about $7^{\circ} \mathrm{C}$ higher than the potato, Solanum tuberosum L., which although closely related to the tomato, is much less liable to chilling injury [44]. Although both species originated from Andean region of South America [45, 46], the cultivated tomato originated from the low altitudes whereas the cultivated potato came from the cooler, high altitudes. Plants which regularly experience temperatures below $10^{\circ} \mathrm{C}$ in their native habitat are expected to be comparatively cold tolerant. Near the Equator, night temperatures are regularly below $10^{\circ} \mathrm{C}$ only at altitudes of $2,000 \mathrm{~m}$ or higher. High-altitude species of Lycopersicon have not contributed to the ancestry of the cultivated tomato and this may explain why the cultivated tomato is less cold tolerant than the potato. Genetic sources of cold tolerance during vegetative growth, however, have been identified among the related wild species and primitive cultivars of tomato [47, 48]. For example, Wolf et al. [48] evaluated cold tolerance of several high altitude accessions of wild tomato species including L. hirsutum (LA 1363, LA 1777) and L. chilense Dun. (LA 1969, LA 1971), and Solanum lycopersicoides Dun. (LA 1964) during vegetative growth and compared that with the cold tolerance of a $L$. esculentum breeding line, UC82B. Chlorophyll fluorescence, electrolyte leakage and plastochron index were used as evaluation criteria. High altitude wild accessions were all more cold tolerant than UC82B. Similarly, Vallejos et al. [49] reported that a high altitude accession of $L$. hirsutum had less growth reduction under a low temperature (day/night $12 / 5^{\circ} \mathrm{C}$ ) compared to a normal temperature regime $\left(25 / 18^{\circ} \mathrm{C}\right)$ than a low altitude $L$. hirsutum or a $L$. esculentum breeding line.

We have initiated a project to investigate the genetic basis of cold tolerance in some of the high altitudinal ecotypes of the wild species L. hirsutum and some genotypes of the cultivated tomato. In a recent investigation, the growth of the parental and reciprocal $F_{1}, F_{2}, F_{3}$ and $\mathrm{BC}_{1}$ generations (a total of 10 generations) of a cross between $L$. esculentum lines UCT5 (cold sensitive) and PI120256 (cold tolerant) were examined under a normal (control) and a low temperature regime. The results indicated that plant absolute growth under low temperature (i.e., absolute cold tolerance) and relative growth (i.e., growth under low temperature relative to growth under normal temperature) (relative cold tolerance) were genetically controlled with simple additive and additive $\times$ additive interactions being the major genetic components (Foolad and Lin, unpublished). These preliminary investigations indicated that cold tolerance of the cultivated tomato could potentially be improved by gene transfer from cold tolerant genotypes. Efforts to identify QTLs for cold tolerance during vegetative growth in an interspecific cross of tomato (L. esculentum $\times L$. hirsutum) is underway.

\section{Relationship between cold tolerance during seed germination and vegetative growth}

Three approaches have been taken to determine the relationship between cold tolerance during germination and vegetative growth. First, an investigation of 31 tomato accessions (plant introductions, breeding lines and cultivars) representing six Lycopersicon species for cold tolerance indicated the presence of a weak correlation $(\mathrm{r}=-0.42, p<0.05)$ between the rate of germination and absolute plant growth under cold stress (Foolad and Lin, unpublished). There was also a weak correlation $(\mathrm{r}=-0.47, p<0.01)$ between the rate of germination under cold stress and the relative cold tolerance during vegetative growth.

The second approach involved the examination of direct and correlated response to selection for cold tolerance. Briefly, in an $F_{2}$ population of a cross between tomato inbred lines UCT5 (cold sensitive during both germination and vegetative growth) and PI120256 (cold tolerant during both stages) selection was made for rapid germination under cold stress, and the selected $F_{3}$ progeny were evaluated for cold tolerance during both germination and vegetative growth. The results indicated that the selected progeny germinated more rapidly than the unselected progeny under cold stress, suggesting that the selection was effective. The selected progeny also exhibited significantly greater vegetative growth $(19 \%$ more fresh weight) than the unselected progeny under cold stress; this observation suggests the presence of a relationship between seed vigor and plant vigor under cold stress (Foolad and Lin, unpublished). Further inspection, however, indicated that the selected progeny also exhibited greater growth than the unselected progeny under the nonstress (control) condition, suggesting that the superior growth performance of the selected progeny under cold stress could be due to higher plant vigor and not cold tolerance. This suggestion was confirmed by the finding that the physiological (relative) cold tolerance of the selected progeny was only slightly $(6.2 \%)$ greater than the physiological cold tolerance of the unselected progeny.

The overall results indicate the presence of a genetic relationship between seed vigor and plant vigor under cold stress, however, there might be different genes contributing to plant vigor and cold tolerance. Thus, selection for rapid seed germination under cold stress may not be effective in improving plant cold tolerance during vegeta- 
tive growth. Research to compare QTLs contributing to cold tolerance during germination and vegetative growth, as the third approach to determine the relationship between cold tolerance at these two developmental stages, is underway.

\section{Relationship between cold and salt tolerance during seed germination}

Three approaches were taken to determine the genetic relationship between cold tolerance and salt tolerance during germination. First, 30 tomato accessions representing six Lycopersicon species were evaluated for germination under cold stress $\left(\mathrm{T}=11^{\circ} \mathrm{C}\right)$ and salt stress $\left(\mathrm{T}=20^{\circ} \mathrm{C}, 150 \mathrm{mM} \mathrm{NaCl}+15 \mathrm{mM} \mathrm{CaCl}_{2} ; \Psi=-850 \mathrm{kPa}\right)$ conditions. The results indicated that most accessions responded similarly to cold and salt stress (i.e., they were equally sensitive or tolerant to both stresses), however, several accessions germinated significantly more rapidly under one stress than the other. For example, some accessions germinated more rapidly under cold than salt stress, whereas others germinated more rapidly under salt than cold stress [40]. Across all genotypes, however, a positive phenotypic correlation was observed between germination under cold and salt stress $(\mathrm{r}=0.77, p<0.01)$. This correlation suggests that some of the same genetic factors which facilitate rapid germination under cold stress also contribute to rapid germination under salt stress, but additional components may also be involved with germination under specific stress conditions.

in the second approach, seeds of $F_{2}$ progeny of a cross between the breeding line UCT5 (sensitive to cold and salt stress during germination) and accession PI120256 (tolerant to both stresses during germination) were evaluated for germination separately under cold stress and salt stress, and in each treatment the fastest germinating seeds (the first $5 \%$ germinated) were identified. The selected seedlings were grown to maturity and self-pollinated to produce selected $F_{3}$ progeny. The selected $F_{3}$ progeny from each experiment were evaluated for germination under cold and salt stress and their performance compared with that of unselected $\mathrm{F}_{3}$ progeny [50]. The results indicated that selection under either cold or salt stress significantly improved progeny seed germination under both cold and salt stress (Table 2). Analysis of response and correlated re-

Table 2 The percentage improvement in germination (response to selction) in the control, cold stress and salt stress treatments ${ }^{a}$

\begin{tabular}{lccc}
\hline \multirow{2}{*}{$\begin{array}{l}\text { Treatment during } \\
\text { selection }\end{array}$} & \multicolumn{3}{c}{ Treatment during progeny evaluation } \\
\cline { 2 - 4 } & Control & Cold stress & Salt stress \\
\hline Control & 4.2 & 4.7 & 5.7 \\
Cold stress & $13.8^{* *}$ & $19.1^{* *}$ & $23.8^{* *}$ \\
Salt stress & $10.8^{* *}$ & $29.0^{* *}$ & $22.5^{* *}$
\end{tabular}

${ }^{a}$ Selection response was calculated as the difference in germination mean between the selected and unselected populations and transformed into percentage relative to germination mean of the unselected population.

${ }^{* *}$ Significant at $p \leq 0.01$. sponse to selection indicated the presence of a significant genetic correlation $(r=0.66-1.00)$ between germination under cold stress and salt stress. These results supported the earlier suggestion of the presence of genes which facilitate rapid germination under both cold and salt stress.

In the third approach, using a $\mathrm{BC}_{1} \mathrm{~S}_{1}$ population of an interspecific cross between $\mathrm{NC} 84173$ (sensitive to both cold and salt stress during germination) and LA722 (tolerant to both stresses during germination), QTLs for cold tolerance during germination were compared with QTLs for salt tolerance during germination for co-localization. The results indicated the presence of some major QTLs on chromosome 1 of tomato which contributed to rapid germination under both cold and salt stress (Table 1, Fig. 2). These QTLs, therefore, were considered to represent germination-related, stress-nonspecific genes [51]. In addition, several QTLs were identified which affected germination under either cold or salt stress; these QTLs were considered to represent germination-related, stress-specific genes. The stress-nonspecific QTLs, however, generally exhibited larger effects than stress-specific QTLs. The results of the QTL analysis were consistent with the observation of a positive family correlation $(r=0.67$, $p<0.01$ ) between germination under cold and salt stress in the $\mathrm{BC}_{1} \mathrm{~S}_{1}$ population and thus, confirmed the earlier suggestion of the presence of a genetic relationship between germination under cold and salt stress.

The overall results indicate the presence of genes which commonly affect germination under both cold and salt stress in addition to genes which may be stress-specific and affect germination only under specific stress conditions. In practice, therefore, either phenotypic or markerassisted selection for rapid germination under a single stress treatment should result in progeny with improved germination under different stress conditions. Whether such a relationship between cold tolerance and salt tolerance also exists at later stages of plant development in tomato is unknown.

\section{Relationship between seed germination under stress and nonstress conditions}

Previous germplasm evaluations indicated that generally tomato seeds that germinate rapidly under nonstress conditions also tend to germinate rapidly under either cold or salt stress. For example, from the data reported by Scott and Jones (1982) [38] we computed a significant phenotypic correlation $(\mathrm{r}=0.75, p<0.01)$ between germination in the control $\left(\mathrm{T}=20^{\circ} \mathrm{C}\right)$ and cold stress $\left(\mathrm{T}=10^{\circ} \mathrm{C}\right)$ treatments for 36 accessions of cultivated and wild tomatoes. Similarly, significant phenotypic correlations $(\mathrm{r}=0.58-$ $0.62, p<0.01$ ) were reported for tomato seed germination under control and salt stress [25, 27, 41]. These results are consistent with the suggestion that similar physiological mechanisms contribute to rapid germination under stress and nonstress conditions [52]. However, the genetic relationship between germination under stress and nonstress conditions had not been previously investigated.

We examined the genetic relationship between tomato 
seed germination under stress and nonstress conditions by three approaches. First, evaluation of 30 tomato accessions for germination under nonstress and cold and salt stress indicated the presence of positive phenotypic correlations between germination under control and cold stress $(\mathrm{r}=0.89, p<0.01)$ and control and salt stress $(\mathrm{r}=0.63$, $p<0.01)$. These correlations suggest the contribution of similar genetic factors to tomato seed germination under stress and nonstress conditions. Notably, however, in this and previous germplasm evaluations $[25,38]$ the magnitudes of the correlation coefficients for germination under stress and nonstress conditions were fairly moderate. A common observation in most of these studies was that some accessions that germinated rapidly under nonstress conditions exhibited poor germination under stress, and vice versa. This observation suggests the presence of genes which might be stress-specific and contribute to rapid seed germination only under specific stress conditions.

In the second approach to determine the relationship between germination under stress and nonstress conditions, an $F_{2}$ population of a cross between UCT5 (slow germinating) and PI120256 (fast germinating) was evaluated for seed germination under either control (nonstress) or stress (cold or salt stress) conditions and in each treatment selection was made for rapid germination. The selected $F_{3}$ progeny from each treatment were then evaluated for germination under both stress and nonstress conditions. Selection for rapid germination under either of the two stresses (cold or salt stress) resulted in progeny with improved germination under both stress and nonstress conditions (Table 2). However, selection for rapid germination under nonstress conditions did not significantly improve progeny seed germination under either of the three (nonstress, cold and salt stress) conditions (Table 2). The latter observation was suggested to be due to the lack of sufficient variation in germination time under nonstress (control) conditions [50]. The overall results, however, indicate the presence of genes which facilitate rapid seed germination under both stress and nonstress conditions.

In the third approach, QTLs for germination time under stress and nonstress conditions were compared with each other for co-localization. The results confirmed the presence of genetic relationship between the rate of germination under stress and nonstress conditions: a few major QTLs were identified which contributed to rapid germination under both stress and nonstress conditions (Table 1, Fig. 2). However, several QTLs were also identified which affected germination either under cold stress or salt stress but not under nonstress conditions. The overall results indicate the presence of genes which commonly affect seed germination under both stress and nonstress conditions in addition to genes which may be stress-specific and affect germination only under specific stress conditions.

\section{Conclusions}

Contrary to the old quantitative genetic theory that quantitative traits are controlled by many loci with small individual phenotypic effects $[15,16,53]$, our QTL mapping investigations indicated that quantitative variation for salt tolerance and cold tolerance in tomato are mainly controlled by the effects of a few major QTLs which act in concert with a number of smaller effect QTLs. Furthermore, most of the identified QTLs exhibited independent effects with little or no epistatic interactions. The results also indicated the presence of little or no genetic correlation between stress tolerance at different developmental stages. Finally, the identification of QTLs which contribute to rapid tomato seed germination under both stress and nonstress conditions should facilitate the development of cultivars with the ability to germinate rapidly in both stress and nonstress environments. The overall results indicated the great utility of molecular marker technology for characterizing the genetic basis of plant response to environmental stresses and for developing stress tolerant genotypes.

\section{Acknowledgements}

I thank Professors Richard Craig and Dennis Decoteau for critical reviewing of the manuscript and Dr. F. Chen and Mr. G. Lin for technical contributions. This research was supported in part by grants from the $\mathrm{Na}$ tional Research Initiative Competitive Grants Program, U.S. Department of Agriculture (\#9600568), and by the College of Agricultural Sciences, the Pennsylvania State University. This is contribution 389 of the Department of Horticulture, the Pennsylvania State University.

\section{References}

[1] Duvick, D.N., 1996. Crop Sci., 36: 539-548.

[2] Dudal, R., 1976. Inventory of major soils of the world with special reference to mineral stress. Plant Adaptation to Mineral Stress in Problem Soils. Cornell Univ. Agric. Exp. Stn., Ithaca, N. Y.

[3] Levitt, L., 1980. Responses of Plants to Environmental Stresses: Chilling, Freezing and High Temperature Stresses. Academic Press, New York.

[4] Munns, R., 1993. Plant Cell and Environm., 16: 15-24.

[5] Bohnert, H.J., Nelson, D.E., Jensen, R.G., 1995. Plant Cell, 7: 1099-1111.

[6] Blum, A., 1988. Plant breeding for stress environment. CRC Press, Boca Raton, Florida.

[7] Foolad, M.R., 1996. Plant Breed., 115: 245-250.

[8] Foolad, M.R., 1997. Plant Breed., 116: 53-58.

[9] Dvorak, J., Epstein, E., Galvez, A., Gulick, P., Omielan, J.A., 1992. Genetic basis of plant tolerance of soil toxicity. In "Plant Breeding in the 1990s" (ed. by Stalker, H.T. and Murphy, J.P.), pp. 201-217. C.A.B. International, North Carolina State University, Raleigh, NC.

[10] Levitt, J., 1980. Responses of Plants to Environmental Stresses: Water, Salt and other Stresses. Academic Press, New York.

[11] Foolad, M.R., 1996. J. Amer. Soc. Hort. Sci., 121: 10061011.

[12] Greenway, H. and Munns, R., 1980. Ann. Rev. Plant Physiol., 31: 149-190.

[13] Johnson, D.W., Smith, S.E. and Dobrenz, A.K., 1992. Theor. Appl. Genet., 83: 833-838. 
[14] Foolad, M.R., Lin, G.Y., 1997. Plant Breed., 116: 363-367.

[15] Mather, K., Jinks, J.L., 1971. Biometrical Genetics. Chapman and Hall, London.

[16] Falconer, D.S., 1989. Introduction to Quantitative Genetics. Longman Scientific and Technical, London.

[17] deVicente, M.C., Tanksley, S.D., 1993. Genetics, 134: 585596.

[18] Zhang, Q., Gao, Y.J., Yang, S.H., Ragab, R.A., Maroof, M.A.S., Li, Z.B., 1994. Theor. Appl. Genet., 89: 185-192.

[19] Li, Z.K., Pinson, S.R.M., Park, W.D., Paterson, A.H., Stansel, J.W., 1997. Genetics 145: 453-465.

[20] Paterson, A.H., Damon, S., Hewitt, J.D., Zamir, D., Rabinowitch, H.D., Lincoln, S.E., Lander, E.S., Tanksley, S.D., 1991. Genetics, 127: 181-197.

[21] Stuber, C.W., Lincoln, S.E., Wolff, D.W., Helentjaris, T., Lander, E.S., 1992. Genetics, 132: 823-839.

[22] Foolad, M.R., Chen, F.Q., 1999. Theor. Appl. Genet., in press.

[23] Maas, E.V., 1986. Appl. Agri. Res., 1: 12-26.

[24] Jones, R.A., 1986. Acta Horticulturae, 190: 101-114.

[25] Jones, R.A., 1986. Euphytica, 35: 576-582.

[26] Foolad, M.R., Lin, G.Y., 1997. Hort Science, 32: 296-300.

[27] Foolad, M.R., Jones, R.A., 1991. Theor. Appl. Genet., 81: 321-326.

[28] Foolad, M.R., Jones, R.A., 1992. Crop Sci., 32: 439-442.

[29] Foolad, M.R., Jones, R.A., 1992. Theor. Appl. Genet., 83: 360-366.

[30] Foolad, M.R., Jones, R.A., 1993. Theor. Appl. Genet., 87: 184-192.

[31] Foolad, M.R., Stoltz, T., Dervinis, C., Rodriguez, R.L., Jones, R.A., 1997. Mol. Breed., 3: 269-277.

[32] Foolad, M.R., Chen, F.Q., Lin, G.Y., 1998. Theor. Appl. Genet., 97: 1133-1144.

[33] Lincoln, S., Daly, M., Lander, E., 1992. Constructing Genetic Maps with MAPMAKER/EXP 3.0. Whitehead Institute Technical Report.
[34] Nelson, J.C., 1997. Mol. Breed., 3: 239-245.

[35] Lyon, C.B., 1941. Bot. Gaz., 103: 107-122.

[36] Tal, M., Shannon, M.C., 1983. Aust. J. Plant Physiol., 10: 109-117.

[37] Shannon, M.C., Gronwald, J.W., Tal, M., 1987. J. Amer. Soc. Hort. Sci., 112: 416-423.

[38] Scott, S.J., Jones, R.A., 1982. Euphytica, 31: 869-883.

[39] Smith, P.G., Millet, R.H., 1964. Proc Amer. Soc. Hort. Sci. 84: 480-484.

[40] Foolad, M.R., Lin, G.Y., 1999. Plant Breed., in press.

[41] Foolad, M.R., Lin, G.Y., 1998. Plant Breed., 117: 171-176.

[42] Foolad, M.R., Chen, F.Q., Lin, G.Y., 1998. Molec. Breed., 4: 519-529.

[43] Lyons, J.M., 1973. Ann. Rev. Plant Physiol., 24: 445-466.

[44] Went, F.W., 1957. The Experimental Control of Plant Growth. Ronald Press Co., New York.

[45] Jenkins, J.A., 1948. The origin of the cultivated tomato. Econ. Bot. 21: 379-392.

[46] Correll, D.S., 1962. The Potato and its Wild Relatives. Texas Research Foundation, Renner.

[47] Patterson, B.D., Paull, R., Smillie, R.M., 1978. Aust J. Plant Physiol., 5: 609-617.

[48] Wolf, S., Yakir, D., Stevens, M.A., Rudich, J., 1986. J. Amer. Soc. Hort. Sci., 111: 960-964.

[49] Vallejos, E., Lyons, J.M., Breidenbach, R.W., Miller, M.F., 1983. Planta, 159: 487-496.

[50] Foolad, M.R., Hyman, J.R., Lin, G.Y., 1999. Plant Breed., in press.

[51] Foolad, M.R., Lin, G.Y., Chen, F.Q., 1999. Plant Breed., in press.

[52] Bradford, K.J., 1995. Water relations in seed germination. In "Seed Development and Germination" (ed. by Kigel, J. and Galili, G.), pp. 351-396. Marcel Dekker, Inc., New York.

[53] Lande, R, 1983. Heredity, 50: 47-65. 